\title{
Bile acid induced colonic irritation stimulates intracolonic nitric oxide release in humans
}

\author{
F Casellas, M Mourelle, M Papo, F Guarner, M Antolín, J R Armengol, J-R Malagelada
}

\begin{abstract}
Aim-To measure the intracolonic release of nitric oxide end products (nitrates plus nitrites) and eicosanoids in response to intraluminal irritation with deoxycholic acid (DCA).

Patients-Seven patients with irritable bowel syndrome.

Methods-The left colon was perfused with a solution with or without $3 \mathrm{mM}$ deoxycholic acid. Aspirates were assayed for eicosanoids by specific radioimmunoassay, and for nitrates plus nitrites by the Griess reaction. To confirm that stimulated colonic mucosa can produce nitric oxide (NO), ancillary studies were performed in vitro using samples of normal mucosa obtained from five surgically resected colons. Samples were incubated for 30 minutes in Kreb's solution, $3 \mathrm{mM}$ DCA or DCA with $1 \mathrm{mM}$ L-nitro-argininemethyl-ester (L-NAME) to inhibit the NO synthase. Finally, NO synthase activity was measured in five samples of human colonic mucosa.
\end{abstract}

Results-Intracolonic release of nitrates plus nitrites was basally undetectable in six of seven patients. Bile acid considerably increased the release of prostaglandin $E_{2}$ and nitrates plus nitrites $(p<0 \cdot 01)$. By contrast, no increase in thromboxane and leukotriene was seen. In vitro mucosal incubation with DCA increased the production of NO synthase products, which was blocked by L-NAME. Activity of $\mathrm{Ca}^{++}$ independent NO synthase was detectable in four of five samples of human colonic mucosa.

Conclusion-The human colonic mucosa responds to bile acid induced irritation by a surge in $\mathrm{NO}$ generation via $\mathrm{NO}$ synthase. (Gut 1996; 38: 719-723)

Keywords: nitric oxide synthase, colonic irritation, colonic perfusion, bile acids.

Digestive System

Research Unit,

Hospital General Vall

d'Hebron, Barcelona,

Spain

F Casellas

M Mourelle

M Papo

F Guarner

M Antolín

J R Armengol

J-R Malagelada

Correspondence to: Dr F Casellas, Digestive System Research Unit, Hospital General Vall

d'Hebron, Pso Vall

d'Hebron 119 ,

Barcelona - 08035, Spain.

Accepted for publication

7 December 1995 production, increased mucosal permeability, and damage of the epithelial surface. ${ }^{12}$ The precise mechanisms that mediate the mucosal response to intraluminal irritation are still not completely understood. Colonic mucosal production of eicosanoids increases after exposure to deoxycholic acid (DCA). ${ }^{3}$ However, inhibition of either cyclooxygenase or thromboxane synthase not only fails to protect the mucosa from the effects of bile acids but even worsens the colonic damage, ${ }^{4}$ suggesting that non-prostaglandin mechanisms also participate. Lipooxygenase metabolites, such as the sulphidopeptidoleukotrienes, may also take part as these substances stimulate water secretion. ${ }^{5}$ We have also shown that human intrajejunal infusion of DCA increases intraluminal release of leukotriene $\mathrm{C}_{4} \cdot{ }^{6}$

It has been shown that bile acids increase the production of reactive oxygen in colonic mucosal scrapings and isolated colonic crypts. ${ }^{7}$ The degradation substances produced by the peroxidative process initiated by oxygen radicals can diffuse away causing oedema, increasing vascular permeability and inflammation. ${ }^{8}$ Nitric oxide (NO) may contribute to the actions of oxygen superoxide annion radical by forming peroxynitrite, which is decomposed into the potent oxidants $\mathrm{OH}^{\cdot}$ and $\mathrm{NO}_{2} \cdot{ }^{9} \mathrm{NO}$ mediates many biological actions, such as smooth muscle relaxation, neurotransmission, inflammation or cytotoxicity. ${ }^{1011}$ However, the possible role of NO in the colonic mucosal response to bile acid induced irritation has not been previously explored.

To investigate whether stimulation of NO synthesis participates in the response of colonic mucosa to bile acid irritation, we measured by a perfusion technique in humans the intracolonic release of $\mathrm{NO}$ derived products before and after colonic infusion of DCA in a group of patients with irritable bowel syndrome.

\section{Methods}

\section{SUBJECTS}

Perfusion studies were performed in seven patients (two men and five women, age ranging from 18 to 46 years) with irritable bowel syndrome. Diagnosis was based on standard clinical criteria (pain ceased after bowel movement, looser stools at onset of pain, more frequent bowel movements at onset of pain, abdominal distension, mucus per rectum, and feeling of incomplete emptying). Routine laboratory tests, upper gastrointestinal barium meal, and colonoscopic examination were normal in every subject. The study was approved by the Institutional Review Board, and all patients gave informed consent.
PERFUSION PROCEDURE

Steady state intracolonic release of nitrates plus nitrites was measured by a double lumen colonic perfusion technique. After ' an 
overnight fast, a double lumen polyvinyl assembly was placed into the descending/ sigmoid colon by sliding it over a guiding wire placed by prior colonoscopy. The orad lumen opened at $50 \mathrm{~cm}$ from the anal verge and was used for infusion of the perfusion solutions. The caudad lumen opened $30 \mathrm{~cm}$ caudally from the orad one and was used to recover the perfusates by siphonage. The correct position of the perfusion tube was assessed by fluoroscopy. Perfusion solutions were infused at $5 \mathrm{ml} / \mathrm{min}$ using a volumetric pump (IMED 927, Milton Trading Estate, Abingdon, UK).

The perfusion solution was an isotonic and neutral solution composed of $280 \mathrm{mmol} / \mathrm{l}$ mannitol and $2 \mathrm{~g} / 1$ polyethylene glycol 4000 (PEG 4000) as a non-absorbable marker. Irritation was induced by the addition of DCA (Koch-Light Laboratories, Colnbrook, Bucks, UK) to the solution to a final concentration of $3 \mathrm{mmol} / \mathrm{l}$. This concentration of DCA was chosen because it causes a very mild irritation while inducing a measurable secretory response in the human colon. ${ }^{3}$ The $\mathrm{pH}$ of the solution was adjusted to $7 \cdot 8$ with $0 \cdot 1 \mathrm{~mol} / 1$ $\mathrm{NaOH}$. To prevent colonic water and electrolytes absorption or secretion induced by the solution, the perfusate did not contain glucose or electrolytes.

The perfusate was recovered by siphonage to preclude the local irritation that would otherwise occur with mechanical or manual aspiration.

\section{EXPERIMENTAL DESIGN}

\section{Main studies}

In the seven irritable bowel syndrome patients, two consecutive perfusion sequences each lasting 100 minutes were performed on a given day. Each sequence began with 30 minutes of colonic washing with the solution without DCA, followed by the test perfusion with or without DCA for 70 minutes (30 minute equilibration period and 40 minute test period). Because the secretory effects of DCA infusion on human colon are reversible, ${ }^{3}$ the order of the perfusions was randomised. During each perfusion period, perfusates were continuously collected on ice and pooled at 10 minute intervals.

\section{Ancillary in vitro studies}

To discover if the colonic mucosa produces nitric oxide via NO synthase, samples of histologically normal mucosa obtained from five surgically resected colons for neoplastic disease were incubated for 30 minutes in $10 \mathrm{ml} \mathrm{Kreb's}$ solution at $37^{\circ} \mathrm{C}$. One aliquot of the sample, which served as control, was incubated in Kreb's solution; a second aliquot of the sample was incubated in Kreb's solution with $3 \mathrm{mM}$ DCA, and a third aliquot of the sample with DCA and $1 \mathrm{mM}$ L-nitro-arginine-methyl-ester (L-NAME, Sigma, St Louis, MO), an inhibitor of the NO synthase. The weight of tissue used in each incubation ranged from 310 to $520 \mathrm{mg}$.
To investigate whether the perfusion of DCA increases nitrates plus nitrites production by stimulation of the bacteria residing in the colon, we performed additional studies incubating human faecal samples with DCA. Faecal samples from subjects without intestinal disease and who had not received any antibiotic treatment were diluted 1:15 in saline and incubated with $3 \mathrm{mM} \mathrm{DCA}$ at $37^{\circ} \mathrm{C}$. Nitrates plus nitrites were determined in aliquots at time 0 and sequentially up to two hours of incubation.

NO synthase activity was measured in mucosal samples from five patients undergoing colectomy for neoplasm. Specimens were always obtained from the distal margin of resection and in every instance, the margin was macroscopically and microscopically free of tumour.

\section{ANALYTICAL PROCEDURES}

Aliquots of $1 \mathrm{ml}$ were stored at $-20^{\circ} \mathrm{C}$ for later analysis of PEG, nitrates plus nitrites and eicosanoids. To prevent in vitro prostanoid synthesis, indomethacin (Sigma) was added to some aliquots up to a concentration of 50 $\mu \mathrm{g} / \mathrm{ml}$.

Samples were assayed for PEG by the Hyden method. ${ }^{12}$ Nitrates were reduced to nitrites using a copper plated cadmium column, and nitrites were determined by the Griess method as previously described. ${ }^{13}$ Results are expressed as nitrites over nitrates $\left(\mathrm{NO}_{2} / \mathrm{NO}_{3}\right)$. Prostaglandin $\mathrm{E}_{2}\left(\mathrm{PGE}_{2}\right)$, thromboxane $\mathrm{B}_{2}\left(\mathrm{TXB}_{2}\right)$, and leukotriene $\mathrm{B}_{4}\left(\mathrm{LTB}_{4}\right)$ were analysed by a previously validated specific radioimmunoassay. ${ }^{14}$

NO synthase activity was assayed in homogenates of the surgical samples of colonic mucosa as the transformation of $\mathrm{L}$-arginine to L-citrulline. ${ }^{15}$ Aliquots of tissue homogenates were incubated in a medium containing 50 $\mathrm{mM}$ potassium phosphate buffer $(\mathrm{pH} 7 \cdot 2), 60$ $\mathrm{mM}$ valine, $1.2 \mathrm{mM}$ citrulline, $120 \mu \mathrm{M}$ NADPH, $24 \mu \mathrm{M}$ arginine, $150000 \mathrm{dpm}{ }^{14} \mathrm{C}$ arginine, $1.2 \mathrm{mM} \mathrm{MgCl} 2,0.24 \mathrm{mM} \mathrm{CaCl}_{2}$ for 10 minutes at $37^{\circ} \mathrm{C}$. The reaction was stopped by dilution and removal of substrate by the addition of $50 \%$ Dowex $50 \mathrm{~W}$ resin mix (200-400 mesh, $8 \%$ cross linked, $\mathrm{Na}^{+}$form). The amount of ${ }^{14} \mathrm{C}$-citrulline formed was measured by scintillation counting. Aliquots with $1 \mathrm{mM}$ L-NAME were used to subtract background citrulline formation, and addition of $1 \mathrm{mM}$ EGTA to specific aliquots served to identify $\mathrm{Ca}^{2+}$ dependent activity. Protein content in the eluates was measured using a BCA protein assay from Pierce (Rockford, IL). Enzymatic activity was expressed as pmol of citrulline formed per $\mathrm{mg}$ of protein per minute.

CALCULATIONS AND STATISTICAL METHODS Results are expressed as mean (SEM). Results of $\mathrm{NO}_{2} / \mathrm{NO}_{3}$ and eicosanoids are expressed as rates of intracolonic release. Release rates were calculated by a standard formula using PEG as a non-absorbable marker. Data correspond to 
Figure 1: Individual values of intracolonic release of $\mathrm{NO}_{2} / \mathrm{NO}_{3}$ before and after DCA stimulation. Basal release was undetectable in six patients and bile acid irritation significantly increased the release of $N O$ breakdown products in five of seven patients.

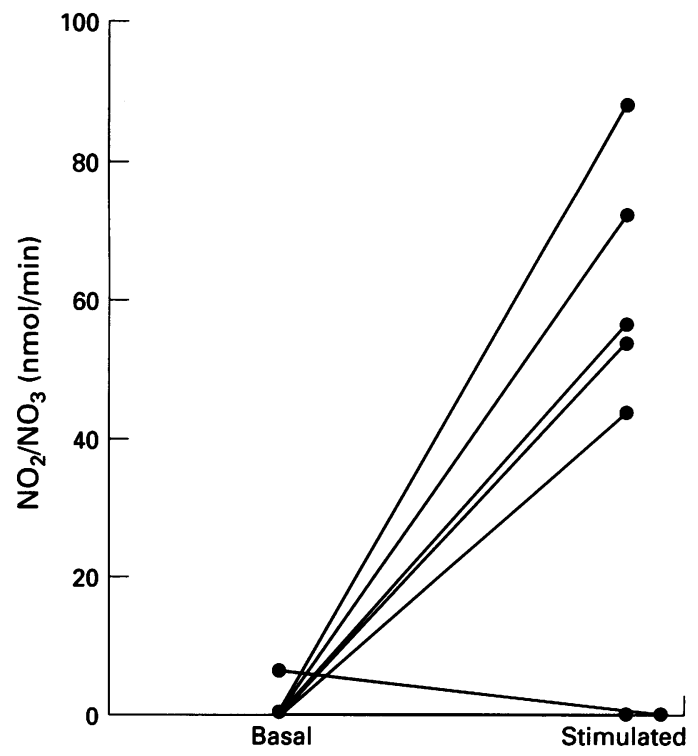

the 20 to 40 minute period from control and DCA perfusion. Statistical differences in colonic perfusion studies were calculated for paired data by the Student's $t$ test. In the in vitro studies the Mann-Whitney $U$ test was used.

\section{Results}

EFFECTS OF DCA ON COLONIC $\mathrm{NO}_{2} / \mathrm{NO}_{3}$ RELEASE

Basal intracolonic nitric oxide release measured as luminal $\mathrm{NO}_{2} / \mathrm{NO}_{3}$ release was undetectable in six of seven patients, and barely detectable in one. As Figure 1 shows DCA perfusion considerably stimulated colonic release of $\mathrm{NO}_{2} / \mathrm{NO}_{3}$ in five patients. For the whole group of seven patients, the mean intracolonic release of $\mathrm{NO}_{2} / \mathrm{NO}_{3}$ was of $0.9(0.9)$ and $45.2(12) \mathrm{nmol} / \mathrm{min}$ before and after DCA stimulation respectively, $\mathrm{p}<0.01$.

\section{EFFECTS OF DCA ON COLONIC EICOSANOID RELEASE}

Eicosanoids were continuously released into the colonic lumen at detectable values under basal conditions. The mean basal release of $\mathrm{PGE}_{2}, \mathrm{TXB}_{2}$, and $\mathrm{LTB}_{4}$ was $0.89(0 \cdot 1)$ $\mathrm{ng} / \mathrm{min}, 0.98(0.2) \mathrm{ng} / \mathrm{min}$, and $0.75(0.1)$ $\mathrm{ng} / \mathrm{min}$ respectively.

Figure 2 represents the change in $\mathrm{PGE}_{2}$ release induced by DCA perfusion. DCA increased intracolonic $\mathrm{PGE}_{2}$ release to 4.98 $(1 \cdot 1) \mathrm{ng} / \mathrm{min}(\mathrm{p}<0.01 v$ basal) with a mean response index (response index $=$ (stimulated-basal)/basal) of $5 \cdot 1$ (1). The mean intracolonic release of $\mathrm{TXB}_{2}$ during stimulation with DCA was $1.76(0.4) \mathrm{ng} / \mathrm{min}$ (Fig 2), which was not significantly different from the basal release, and represents a response index of only $1 \cdot 2$ $(0 \cdot 4)$. Similarly, intracolonic release of $\mathrm{LTB}_{4}$ with DCA stimulation was $1 \cdot 1(0 \cdot 1) \mathrm{ng} / \mathrm{min}$ (Fig 2), which was not significantly different from basal release, and represents a response index of only $0.9(0.5)$.
EFFECT OF DCA ON IN VITRO COLONIC MUCOSAL PRODUCTION OF NO DERIVED METABOLITES

Mean concentration of $\mathrm{NO}_{2} / \mathrm{NO}_{3}$ in supranatants of control incubations of human colonic mucosa was $2.6(0.3) \mathrm{nmol} / \mathrm{ml}$. As Figure 3 shows, incubation of mucosal samples
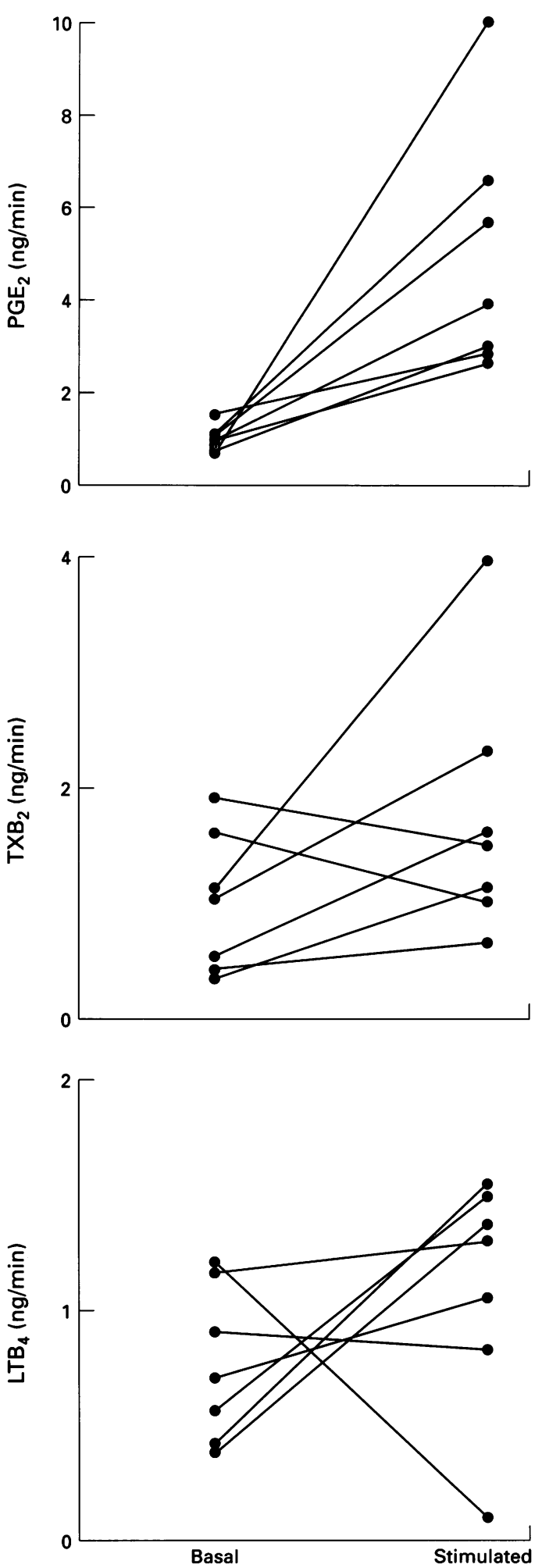

Figure 2: Individual values of intracolonic release of $P G E_{2}$, $T X B_{2}$, and $L T B_{4}$ before and after DCA stimulation. All three eicosanoids were released at detectable values under basal conditions. $P G E_{2}$ release into the colonic lumen was considerably increased with $D C A$ perfusion (response index of $5 \cdot 1, p<0 \cdot 01)$. Intraluminal release of $T X B_{2}$ was only slightly stimulated by $D C A$ (response index of $1 \cdot 2, N S$ ). $L T B_{4}$ release was not stimulated by $D C A$ (response index of $0.9, N S$ ). 


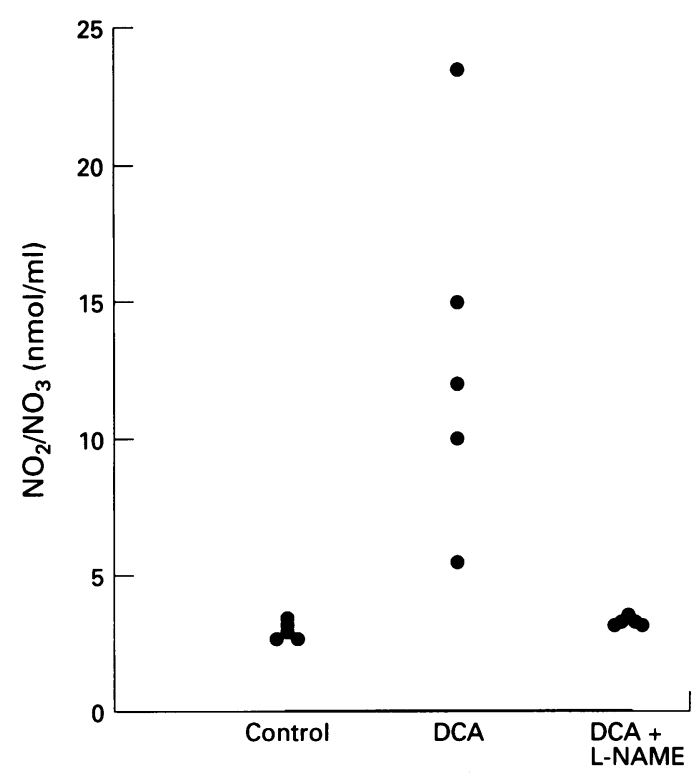

Figure 3: Individual values of in vitro production of $\mathrm{NO}_{2} / \mathrm{NO}_{3}$ by samples of human colonic mucosa. The production of $\mathrm{NO}_{2} / \mathrm{NO}_{3}$ was increased in the presence of $D C A$, but the addition of L-NAME inhibited the response to $D C A$.

with DCA increased the concentration of $\mathrm{NO}_{2} / \mathrm{NO}_{3}$ in the medium $(13.2(3 \cdot 0) \mathrm{nmol} / \mathrm{ml}$, $\mathrm{p}<0.01 v$ control). However, in the presence of L-NAME, addition of DCA did not change $\mathrm{NO}_{2} / \mathrm{NO}_{3}$ concentrations $(3 \cdot 14(0 \cdot 1) \mathrm{nmol} / \mathrm{ml}$, $\mathrm{p}<0.01 v$ DCA stimulated values).

Incubation of diluted faecal samples with DCA did not show any generation of $\mathrm{NO}_{2} / \mathrm{NO}_{3}$, which was undetectable both in basal aliquots and in the sequential determinations up to two hours of incubation. These data suggest that DCA did not stimulate the production of nitrate plus nitrite by bacterial populations present in the colonic lumen.

Total citrulline formation by homogenates of human colonic mucosa was $4.70(0.64)$ $\mathrm{pmol} / \mathrm{mg} / \mathrm{min}$; in the presence of EGTA, the amount of citrulline formed was $4.66(0.72)$, and in the presence of L-NAME, $4 \cdot 15(0 \cdot 60)$ $\mathrm{pmol} / \mathrm{mg} / \mathrm{min}$. Thus, activity of the $\mathrm{Ca}^{2+}$ dependent fraction of the NO synthase was undetectable in most samples of colonic mucosa. In contrast, the $\mathrm{Ca}^{2+}$ independent fraction was detectable in four of five samples (median $0.12 \mathrm{pmol} / \mathrm{ml} / \mathrm{min}$, range 0.00 to $0 \cdot 15)$.

\section{Discussion}

Our colonic perfusion studies show that under basal conditions release of $\mathrm{NO}_{2} / \mathrm{NO}_{3}$ into the human colonic lumen is undetectable by the Griess reaction. However, after mild irritation with a weak dihydroxy bile acid solution, the release of NO metabolites noticeably increases in most subjects. In addition, irritation induced the release of $\mathrm{PGE}_{2}$ into the colonic lumen.

It is known that $\mathrm{PGE}_{2}$ has cytoprotective properties in the colonic mucosa, preventing damage induced by toxin producing clostridia, ethanol, immune complexes or trinitrobenzenesulphonic acid. ${ }^{16} 17$ In vitro studies have shown that $\mathrm{PGE}_{2}$ exerts a direct protective action on duodenal luminal cell membrane vesicles incubated with deoxycholate. ${ }^{17}$ These findings suggest that the mucosal eicosanoid response to intraluminal irritants is a protective response. Nearly all human cells can metabolise arachidonic acid to prostaglandins, and it has been shown that intestinal epithelial cells produce $\mathrm{PGE}_{2}{ }^{18}$

The source of the intracolonic $\mathrm{NO}_{2} \mathrm{NO}_{3}$ measured in our study is unlikely to be other than the colonic mucosa. Conceivably, ingested food particles could be a complementary source but the absence of nitrates and nitrites during the basal perfusion and its presence only after the irritative stimuli would exclude this possibility. To corroborate that the origin of nitrates and nitrites is the NO produced by the colonic mucosal NO synthase, in vitro studies incubating normal colonic mucosa with DCA with or without L-NAME were performed. These ancillary studies provide evidence showing that DCA is able to stimulate the mucosal production of nitrates and nitrites and the response is blocked by L-NAME. This finding suggests that nitrates plus nitrites are generated by the colonic mucosa, and in fact our experiments with mucosal homogenates showed that inducible NO synthase activity is present in human colonic mucosa. In addition, the experiments with faecal samples showed that bacteria present in the colonic lumen did not release $\mathrm{NO}$ at detectable values in response to DCA. However, it is not possible to establish the precise cellular source of $\mathrm{NO}_{2} / \mathrm{NO}_{3}$; epithelial cells and macrophages as well as vascular and neural elements may contribute.

The functions of NO in the gastrointestinal tract are diverse, and include regulation of motility, non-adrenergic, non-cholinergic neural transmission, and preservation of mucosal blood flow. Because the nitrovasodilator NO donors attenuate the endotoxin induced intestinal damage, ${ }^{19}$ it has been suggested that constitutive NO exerts a protective effect on intestinal mucosa. However, the mechanisms of such putative protection have not been elucidated. On the other hand, pretreatment with dexamethasone prevented the delayed changes induced by lipopolysaccharide by inhibiting the induction of calcium independent NO synthase activity. ${ }^{20}$ These results suggested that NO formed by the inducible NO synthase does not have protective properties. Inducible NO synthase generates large amounts of NO with powerful cytotoxic and antimicrobial effects. ${ }^{10}$ Cytotoxicity of neutrophils as well as macrophages is related, at least in part, to generation of NO via an NO synthase induced by endotoxins and cytokines. ${ }^{21-23}$ Thus, we postulate that the NO mediated response to intracolonic irritants seen in our study may represent an active mechanism of mucosal defence by which the mucosa attempts to destroy intraluminal bacteria by releasing NO.

In summary, our studies of human colonic mucosal response to bile acid irritation with in vivo colonic perfusions and in vitro mucosal studies show a mucosal response to irritation entailing increased release of $\mathrm{PGE}_{2}$ and $\mathrm{NO}$ 
breakdown products. This response may represent an attempt to clear the intraluminal irritants releasing cytotoxic NO, and increasing the synthesis of cytoprotective $\mathrm{PGE}_{2}$.

Part of this study was presented at the Annual Meeting of the American Gastroenterological Association, May 1993, Boston, Massachusetts. The authors thank Ms Anna Aparici and Ms Maite Casaus for their help in performing the studies. This work was partly supported by a grant from Fondo de Investigaciones Sanitarias de la Seguridad Social of Spain.

1 Krag E, Phillips SF. Effect of free and conjugated bile acids on net water, electrolyte, and glucose movement in the 2 Mekhiian HS, Phillips SF, Hofmann AF. Colonic secretion of water and electrolytes induced by bile acids: perfusion of water and electrolytes induced by bile acids:
studies in man. $\mathcal{f}$ Clin Invest 1971; 50: 1569-77.

3 Rampton DS, Breuer NF, Vaja SG, Sladen GE, Dowling RH. Role of prostaglandins in bile acid-induced changes in rat colonic structure and function. Clin Sci 1981; 61: 641-8.

4 Argenzio RA, Henrikson CK, Liacos JA. Effect of prostaglandin inhibitors on bile acid-induced mucosal damage of porcine colon. Gastroenterology 1989; 96: 95-109.

5 Smith PL, Montzka DP, McCafferty GP, Wasserman MA, Fondacaro JD. Effect of sulfidopeptide leukotrienes D4 and $\mathrm{E} 4$ on ileal ion transport in vitro in the rat and rabbit. and E4 on ileal ion transport in vit.

6 Casellas F, Guarner F, Rodríguez R, Malagelada JR. Human jejunal LTC $_{4}$ response to irritant bile acids. Eur $\mathcal{J}$ Gastroenterol Hepatol 1991; 3: 393-7.

7 Craven PA, Pfansiel J, DeRubertis FR. Role of reactive oxygen in bile acid stimulation of colonic epithelial proliferation. $\mathcal{F}$ Clin Invest 1986; 77: 850-9.

8 Southorn PA, Powis G. Free radicals in medicine. I. Chemical nature and biologic reactions. Mayo Clin Proc 1988; 63: 381-9.

9 Beckman JS, Beckman TW, Chen J, Marshall PA, Freeman BA. Apparent hydroxyl radical production by peroxynitrite: implications for endothelial injury from nitric oxide trite: implications for endothelial injury from nitric oxide and superc
10 Moncada S. The L-arginine: nitric oxide pathway. Acta Physiol Scand 1992; 145: 201-27.

11 Lowenstein CJ, Dinerman JL, Snyder SH. Nitric oxide: a physiologic messenger. Ann Intern Med 1994; 120: 227-37.

12 Hyden $\mathrm{S}$. A turbidimetric method for the determination of higher polyethylene glycols in biological materials. Ann $R$ Agric Sweden 1955; 22: 139-45.

13 Guarner C, Soriano G, Tomas A, Bulbena O, Novella MT, Balanzó J, et al. Increased serum nitrite and nitrate levels in patients with cirrhosis. Hepatology 1993; 18: 1139-43.

14 Casellas F, Guarner F, Rodríguez R, Salas A, Tallada N, Malagelada JR. Abnormal jejunal prostanoid release in response to mucosal irritation in chronic ulcerative colitis. Eur 7 Gastroenterol Hepatol 1991; 3: 667-73.

15 Mourelle M, Guarner F, Moncada S, Malagelada JR. The arginine:nitric oxide pathway modulates sphincter of Oddi motor activity in guinea pigs and rabbits. Gastroenterology motor activity in guinea

16 Erickson RA, Chang K, Lifrak E, Rivera N, Stachura J. 16,16 -dimethyl prostaglandin $\mathrm{E}_{2}$ reduces bile acid-mediated intestinal vascular injury in rats. Gastroenterology 1992; 102: 1295-305.

17 Zhao D, Hirst BH. Prostaglandin protects against bile salt induced increases in proton permeation of duodenal brush border membrane. Gut 1991; 32: 645-8

18 Hata $Y$, Ota S, Nagata T, Uehara Y, Terano A, Sugimoto T. Primary colonic epithelial cell culture of the rabbit producing prostaglandins. Prostaglandins 1993; 45: 129 - 11.

19 Boughton BJR, Moncada S. Protective effect of S-nitroso-N-acetylpenicillamine in endotoxin-induced acute intestinal dampenicillamine in endotoxin-induced acute intestin

20 Boughton-Smith NK, Evans SM, Whittle BJR, Moncada S. Dexamethasone inhibits endotoxin-induced vascular permeability and nitric oxide synthase in the rat intestine. $\mathrm{Br}$ f Pharmacol 1992; 107: 79P

21 Hibbs JB, Taintor RR, Vavrin Z, Rachlin EM. Nitric oxide: a cytotoxic activated macrophage effector molecule. Biochem Biophys Res Commun 1988; 7: 87-94.

22 McCall TB, Boughton-Smith NK, Palmer RMF, Whittle BJR, Moncada S. Synthesis of nitric oxide from L-arginine by neutrophils. Release and interaction with superoxide anion. Biochem f 1989; 261: 293-6.

23 Stuehr DJ, Nathan CF. Nitric oxide. A macrophage product responsible for cytostasis and respiratory inhibition in tumor target cells. $\mathcal{F}$ Exp Med 1989; 169: 43-55. 